\title{
Le Picaresque : Analyse Du Roman Chair Piment De Gisele Pineau
}

\author{
Rose Auma \\ Masinde Muliro University of Science and Technology, Kenya
}

Doi:10.19044/esj.2019.v15n14p38～URL:http://dx.doi.org/10.19044/esj.2019.v15n14p38

\section{Résumé}

Cet article s'intéresse à dévoiler le picaresque dans le roman Chair Piment de Gisèle Pineau. Le picaresque est la mise en forme romanesque du picaro. C'est un roman fictif qui raconte la vie d'un personnage misérable vivant en marge de la société, confronté par toutes sortes de difficultés et de péripéties. Ce genre exprime l'esprit, la pensée et la philosophie du héro picaresque, généralement caractérisé par la pauvreté. D'après Souiller, (1980), le picaro dépeint une société en décadence. Chair Piment de Gisèle Pineau est paru en 2002 chez Mercure de France. Le roman raconte la vie de Mina, son enfance à Piment en Guadeloupe, son voyage en France durant sa jeunesse et finalement le retour au pays natal. L'action se déroule en Guadeloupe aux Antilles, puis en France et encore en Guadeloupe. Le personnage principal, révolté, naïf et amère, c'est Mina. Elle incarne le rejet des valeurs sociales. Le récit se situe dans les Antilles. La région est composée majoritairement de noirs d'origine africaine et de métis. Elle comprend aussi les Amérindiens, les Indiens caraïbes, les Hindous et les Blancs. Issus des traditions orales, les écrivains de la Caraïbe ont donné la vie à une littérature écrite en langue française. Celle-ci exprime leurs traditions, leurs cultures, leurs images, mais aussi et surtout la souffrance et la révolte des populations déracinées, déportées de l'Afrique en Amérique.

Mots clés : Picaresque, picaro, pauvreté, révolte. 


\title{
The Picaresque: Analysis of Chair Piment of Gisele Pineau
}

\author{
Rose Auma \\ Masinde Muliro University of Science and Technology, Kenya
}

\begin{abstract}
This article is interested in unmasking the picaresque in the novel Chair Piment of Gisèle Pineau. The picaresque is putting in written a fictitious novel that narrates the life of a miserable character living in the margins of the society. The character experiences all sorts of difficulties and peripeties. The genre expresses the spirit, thoughts and philosophy of the picaresque hero, generally characterized by poverty. Souiller (1980), states that the picaro depicts decadence in the society. Mercure, France first published the novel in 2002. The novel narrates the life of Mina, her childhood in Guadeloupe, her travel to France in her youth and finally the return to her country of birth. The action takes place in Guadeloupe in the Antilles, then in France and finally in Guadeloupe. The main character, rebellious, naïve and bitter is Mina. She portrays lack of moral values. The action is located in the Antilles, a region occupied mainly by blacks, metis, Amerindians, Caribbean Indians, Hindus and whites. With a background of oral tradition, the Caribbean writers developed literature written in French. Through it, they express their traditions, cultures, images as well as the suffering and revolt of people removed from their roots and deported from Africa to America.
\end{abstract}

Keywords: Picaresque, picaro, poverty, revolte.

\section{Introduction}

Le roman picaresque est un genre littéraire né en Espagne au XVIe siècle. Le mot picaresque est dérivé de l'adjectif espagnol « picarresco », créé à partir de la racine espagnole « picaro », (misérable, futé). Le mot picaro est paru pour la première fois en 1525 avec le sens de « marmiton », sans aucun sens péjoratif. Le sens va évoluer et en 1726, le premier Dictionnaire de l'Académie espagnole définit le mot « Picaro » comme « bas, misérable, dolosif, dépourvu d'honneur, sans honte ». Le mot finira par prendre un sens péjoratif. Le picaro se caractérise par la pauvreté. Celui-ci est pauvre de liberté, de vêtements, de nourriture, de volonté et d'intelligence. Il s'agit d'un personnage marginal qui vit au bas de la société. Maurice Molho, (1990), définit le picaro comme «un 
personnage de basse extraction, sans métier fixe, serviteur aux nombreux maîtres, incessant voyageur, vagabond, voleur, mendiant, lâche ».

\section{Genre picaresque}

Le genre picaresque est autobiographique, racontant la vie d'un héro misérable qui vit en marge de la société aux prises avec toutes sortes de difficultés et de péripéties. Ce genre romanesque exprime l'esprit, la pensée et la philosophie du héro picaresque. D'après Didier Souiller, (1980), le picaro dépeint une société en décadence. Ce genre romanesque se développe en France au 18eme siècle. Il présente un héros débrouillard, d'origine modeste qui essaie de sortir de son état. Celui-ci aime l'aventure et fréquente tous les milieux sociaux. Le récit raconte la vie du personnage, dès sa naissance jusqu' à la fin de sa vie du récit. Le roman met en scène plusieurs personnages. Le premier texte romanesque picaresque connu, c'est Lazarillo de Tormes publié en 1554. L'auteur de ce texte traite les thèmes de faim, de représentation de certains types sociaux et de transgression des valeurs sociales de l'époque. D'après Molho, (1990), l'ouvrage de fondation, Guzman d'Alfarache est publié quarante ans après. Celui-ci a toutes les caractéristiques du genre picaresque.

Selon Didier Souiller, (1980), l'intrigue dans un roman picaresque comporte :

- Une naissance ignoble du héros ;

- Une éducation négligée et de mauvais traitements dont il est victime ;

- Thèmes d'errance, d'apparence, de faim, d'amour impossible et du destin.

D’après cet écrivain, le récit est fondé sur quelques règles :

- l'itinéraire géographique (matériel et spirituel);

- le passage par différents maîtres ;

-les récits enchâssés ou les histoires indépendantes.

Il ajoute que le récit picaresque ne finit jamais et que la structure reste ouverte. Enfin, la littérature picaresque implique un certain ton ou style : l'ironie, l'humour, l'autobiographie fictive. C'est un récit de l'antihéros. Le but du picaro est de changer sa condition. Celui-ci fait recours à des moyens bizarres pour s'échapper à la faim, à la pauvreté, à tous les problèmes auxquels il fait face. Il incarne généralement le rejet des valeurs sociales.

\section{2. "Chair Piment » de Gisèle Pineau}

Chair Piment se présente comme un récit autobiographique, qui raconte la vie de Mina, l'héroïne, dès sa naissance. Le récit s'ouvre invariablement sur le récit des origines. La vie du picaro est racontée à partir d'un présent d'où la romancière écrit pour le justifier ou amener le lecteur à juger son existence. Le caractère moralisateur du roman illustre un 
comportement déraisonnable face aux normes de la société. Le comportement de Mina est déraisonnable. L'héroïne emploi des moyens absurdes pour sortir de sa situation d'origine. La mort violente des membres de sa famille, l'un après l'autre, pousse Mina à quitter son pays pour la France, chez sa sœur. Arrivée là, elle ne s'entend pas avec elle et quitte finalement sa maison pour la sienne où elle trouve la liberté de se lancer dans son comportement bizarre. Mina, le personnage fait recours au sexe pour oublier ses problèmes. D'après le narrateur, elle devient une femme à la chasse des hommes pour le sexe, dépourvue de l'honneur.

«Il lui fallait les hommes. Leur masse sur son corps. Leur suer. (...) Elle les chassait comme les légumes aux étals des commerçants. » (p. 93-94)

Le récit est une satire de la société. Le narrateur y présente, de façon ridicule, le comportement de Mina qui est contraire à l'éthique et à la morale. La structure très libre du roman permet à l'auteur d'introduire un épisode après l'autre, sans faire sortir le lecteur de ce qui précède. Ce manque de logique et de nécessité interne dans le développement de l'intrigue dépeint le roman picaresque.

\subsection{Auteur}

Gisèle Pineau, d'origine Antillais est élevée en France. Elle s'installe à Paris pour suivre des études de lettres après son Bac mais abandonne ses études à cause des raisons financières et obtient finalement un diplôme d'infirmière. Son roman Grande drive des esprits reçoit le prix des lectrices de « Elle » en 1994 et un autre, L'Esperance macadam le prix RFO en 1996, Pineau, 2002). Dans Chair Piment, l'auteur raconte une histoire qui tourne autour des Antilles (plus précisément de la Guadeloupe) et de la France. Le récit est situé entre les années soixante-dix et deux mille.

\subsection{Résumé du roman}

Il s'agit de l'histoire d'une jeune fille née dans une famille guadeloupéenne en difficulté. Adorée de ses parents, elle va finalement perdre tous les deux, violemment, et peu après, sa sœur qu'elle aimait, est morte d'un incendie. Elle reste seule avant d'être invitée en France par sa demi-sœur qui y habite avec son mari. Elle a connu des difficultés, la solitude, la frustration et la faim. L'auteur nous présente son enfance heureuse, son adolescence douloureuse et solitaire et une jeunesse et vie adulte libérés de valeurs de la morale. Il s'agit donc d'un personnage qui pourrait être qualifié de picaro, dont le voyage en France pourrait être perçu comme «errance picaresque ou aventure picaresque ». Un personnage révolté, vengeur et qui a des idées méchantes dans la tète. Une héroïne dépourvue de valeurs. L'auteur emploi des techniques narratives picaresques en narrant l'histoire du mariage misérable 
et sans amour des parents de Mina, de la héroïne et la vie en général des habitants de Piment, une ville Guadeloupéenne.

\section{Contexte historique et social du récit}

Le récit se situe dans les Antilles ou les Caraïbes. Il s'agit de la littérature Antillaise (Guyane, Martinique et Guadeloupe). La Guadeloupe se situe dans les Caraïbes, bassin versant de la mer des Caraïbes. La région est composée majoritairement de noirs d'origine africaine et de métis. Issus des traditions orales, les écrivains de la Caraïbe ont écrit une littérature en langue française. Celle-ci exprime leurs traditions, leurs cultures, leurs images, mais aussi et surtout la souffrance, la révolte des populations déracinées, déportées de l'Afrique en Amérique.

La population des Antilles est composée des populations noires dont l'histoire est liée à celle de l'esclavage L'état économique, social et religieux de la Caraïbe, juste comme celui de l'Afrique post colonial favorise l'esprit picaresque. Durant l'esclavage et la colonisation, les rapports entre les blancs et les noirs ont été des relations du dominant et de dominé, maître et serviteur. En réaction, les noirs écrivains démontrent les humiliations, traumatismes et injustices liés à ces rapports noir-blancs. Les noirs sont devenus victimes, prisonniers de la pauvreté, désillusionnés, désespérés et ils ont perdu leurs scrupules. Le narrateur fait référence dans l'œuvre à ces rapports :

Quand mon mulâtre a fini de me considérer, il a demandé :

« Pourquoi tu veux devenir propriétaire, négresse ? (...) Il a lâché son rire qui a craqué au mitan de mon cœur, plus fort que le tonnerre dans le ciel. (...) Vous-mêmes nègres, vous n'avez pas de pays et tu voudrais que tes cabris entrent dans le rang des possédeurs ! Ma parole... » (p. 66)

Il s'agit ici d'un homme, (mulâtre), qui parle à une noire, (négresse), et qui lui rappelle que les nègres n'ont pas de pays. Ceci dépeint un espace clos, interdit, où le «nègre " n'est pas vraiment libre. Ce qui est même étonnant, c'est que celui qui s'adresse à lui n'est qu'un mulâtre. La dame noire voulait acheter un morceau de terre, mais on le lui interdit à cause de la couleur de sa peau. Les rapports entre les noirs et les blancs sont aussi explicités quand l'un des hommes, Christian, essaie de convaincre Mina de se marier avec lui. Il lui demande si elle accepterait de se marier avec lui s'il était noir. Mina lui répond que :

« On couche ensemble et ça s'arrête là. » (p. 95)

Cette phrase nous montre bien qu'il y a toujours des problèmes entre les noirs et les blancs. Il pense que Mina ne veut pas se marier avec lui à cause de la couleur de sa peau. Le sentiment des injustices et les frustrations qui pèsent sur les noirs ont menés aux révoltes chez les jeunes qui ont commencé à défier les lois traditionnelles. Les difficultés économiques ont résulté au 
chômage, à la mendicité, à la délinquance, aux orphelins, aux enfants abandonnés, à l'exode rurale, etc. Ces faits ont influencé la littérature africaine et de la sphère francophone. Dès lors, les écrivains francophones dépeignent des personnages d'une moralité douteuse, des êtres inconsistants, des picaros.

Le roman de Gisèle Pineau se situe entre les années soixante-dix et les années deux mille. Le texte apparaît après le fondement du mouvement de la Créolité vers la fin des années quatre vingt. La créolité est un mouvement fondé sur la langue créole et les traumatismes provoqués par la traite, l'exil et l'esclavage. Notons que l'auteur est Antillaise et que cela aurait peut-être de l'influence sur son œuvre. Les écrivains de l'époque, à travers l'écriture et le langage sont à la quête identitaire entamée par la Négritude et le mouvement de l'Antillanité. L'Antillanité est un concept né à la fin des années soixante à partir du constat selon lequel la société antillaise est malade. Elle souffre d'avoir subi une politique de colonisation réussie. Face à cette observation, les écrivains de l'époque proposent un remède : la quête de l'identité antillaise.

Le texte est une satire de la société. L'auteur nous présente une histoire pleine d'ironie, d'exagérations (haute négresse, etc.) et de ridicule, où elle critique les vices et les comportements aberrants des habitants de Piment. La société décrite dans le texte est caractérisée par des conflits, de l'adultère, de l'inceste et de la sorcellerie, ce qui dépeint une société en décadence. Le surnaturel y règne aussi, comme dans toute autre communauté africaine.

Pour l'étude de cette œuvre, nous optons pour la sociocritique. Ceci nous permettra de dégager du corpus ce qui pourrait être qualifiée de poétique picaresque, mais aussi de passer de la description à la valeur. Nous nous intéressons au fonctionnement interne du texte et au travail de l'écrivain. Les trois éléments principaux sont :

1. L'action : les personnages et leur comportement.

2. L'espace : il s'agit de quel type d'univers ? Quelles sont les stratégies employées par l'auteur pour attirer l'attention du lecteur?

3. Le temps de l'action

\section{L'action}

L'étude des personnages correspond à celui du thème du texte. Chaque personnage incarne un thème spécifique. Parmi les thèmes que nous pouvons retenir de ce roman, il faut noter le racisme ou la relation noir/blanc et le sentiment négrophobe lié à l'histoire des Caraïbes, des mariages misérables, de l'amour, de la dégradation des mœurs, des rapports conflictuels et de la sorcellerie.

\subsection{Mina Monterio}

C'est le personnage principal. Elle est la troisième née de sa famille. Elle perd ses parents ainsi que sa sœur toute jeune. Restée d'abord avec sa 
sœur Rosalia et ensuite seule après la mort de celle-ci d'un incendie, elle a vécu la souffrance, la solitude et la faim. Elle ne va plus à l'école après la mort de son père car elle doit s'occuper de sa sœur ainée. La mort de sa sœur était traumatisante et elle ne cesse pas de la voir enflammée. Celle-ci la hante. Elle voyage finalement en France, à l'âge de quatorze ans à l'invitation de sa demisœur Olga, le seul membre de sa famille encore vivant qui y habite avec son mari. En arrivant en France, Olga, sa demi-sœur l'accueil en disant :

- $\quad$ Tu commences une nouvelle vie », (p. 26)

- Regarde ton lit Mina, tu vois, on t'attendait ! Et maintenant ne pense plus à ce que tu as vécu. Ta nouvelle vie commence aujourd'hui! » ( p. 26)

Il s'agit d'une nouvelle vie différente de celle qu'elle avait menée en Guadeloupe. C'est un autre épisode dans le récit. Cela nous montre que la vie de la jeune fille jusqu'à ce moment-là n'était pas en roses. La sœur de Mina est riche. Ici, l'auteur fait la comparaison entre les deux situations dans lesquelles Mina se trouve, à Piment en Guadeloupe et en France chez sa demisœur. La jeune fille souffreteuse allait bientôt commencer une nouvelle vie dépourvue de la souffrance. Plus de solitude ni de faim.

Mina, personnage qui incarne la dégradation des meurs dans la société finit par séduire et coucher avec le mari de sa demi-sœur qui la logeait, qui la traitait comme son enfant. La sœur qui lui a donné une nouvelle vie, qui l'a reçu chez elle, l'a livré de la souffrance dans laquelle elle est restée après la mort de ses parents. L'auteur raconte comment celle-ci manipule son beau frère jusqu'à ce qu'il couche avec elle. C'est de la parodie.

Elle posa un doigt sur les lèvres de l'homme. Un geste de femme en séduction. (...)

- Non Mina..., essaya-t-il encore.

- Ne dis rien, supplia-t-elle en déboutonnant sa chemise blanche. Je veux ta bouche. (p. 83)

Mina fait cela, non pas parce qu'elle aime Douglas, mais pour venger sa sœur Rosalia, maltraitée par Olga durant son enfance. En plus, celle-ci n'avait pas assisté aux obsèques de Rosalia en dépit du fait qu'elle est riche et pouvait facilement payer son billet d'avion pour y aller. Elle justifie pour quoi elle a séduit son beau-frère après cet acte sexuelle. Elle n'a pas de respect pas sa demi-sœur. D’après le narrateur :

Elle avait envie de la voir blessée (Olga), de la voir terrassée, de lui crier : "Ton Doug, il a couché avec moi ! On était au lit ensemble ! La sous ton toit. J'ai vengé Rosalia, se disait-t-elle pour se justifier. Oui, Olga mérite tout cela. (p. 84)

Coucher avec son beau frère dans la maison de sa sœur c'est avoir du courage excessif, de l'impunité. C'est un acte abominable, inacceptable, 
dépourvu de l'honneur et qui va contre les valeurs de la morale. Elle n'a pas honte, ce qui relève du picaresque. Le narrateur ajoute que :

«Et Mina avait l'impression de n'être rien de moins qu'un ver logé dans le fruit, un serpent lové dans le cœur de sa sœur. » (p. 84)

Juste après cela, elle subi la honte et se sent coupable car Olga ne lui avait jamais fait du mal. Au contraire, elle aimait bien sa petite sœur. Elle sait très bien que ce qu'elle fait est absurde mais elle continue à coucher avec Douglas. On peut donc la qualifiée de personnage picaresque. Finalement, elle quitte la maison de sa sœur pour vivre seule, et elle commence à séduire d'autres hommes.

Ce personnage est libéré des valeurs. La préoccupation principale de Mina pendant qu'elle est en France est de faire le sexe, avec n'importe qui, gratuitement. Faire l'amour lui fait plaisir, lui donne l'illusion de l'amour. (p. 12) Elle le fait aussi pour s'échapper des fantômes qui la hantent et la suivent depuis son départ de la Guadeloupe, surtout celui de sa sœur Rosalia, brulée vive dans un incendie. D’après le narrateur,

«Elle s'ouvrait. Se cabrait. Se laissait tourner et retourner, pénétrer...En redemandait. Voulait les sentir dur en elle. » (p. 17) Et il s'agissait de n'importe qui, tout le monde :

«Des hommes de passage ramassés sur le parking de la cité, entre deux voitures ou détournés de leur train (...). Des célibataires. Des jeunes, des vieux. Des bons maris. Des Noirs, des Blancs, des Arabes...

Ils entraient en elle, gratis, tâtait sa chair, goûtaient sa peau. Fallait qu'elle soit prise. Possédée. Traversée, sans paroles, par des sexes d'hommes. » (p.17)

Le comportement de Mina n'est pas normal. Il s'agit d'une femme rebelle. Toute femme chérit son corps et ne peut pas le donner à n'importe qui, n'importe comment. Mina ne sait même pas avec qui elle avait couché. C'est comme une maladie, une « fièvre » d'après le narrateur, de la folie.

«Elle ne retenait plus leurs noms, non plus leur visages...Ça la prenait comme une fièvre. A ces moments là, elle ne gouvernait plus son corps. Elle consommait du sexe, le sexe dressé des hommes. En redemandait. En rêvait même parfois. ॥ (p. 17)

Dès qu'elle avait eu ce qu'elle voulait, c'était fini, et elle les chassait même de sa maison. Il ne s'agissait pas de la prostitution car elle ne demandait pas de paiement après le sexe, comme elle le dit à l'un des hommes qui voulait la revoir.

- $\quad$ Il faut que tu partes.

- T'es vraiment une drôle de fille...

- Dépêche-toi !

- Appelle-moi (...)

- Je t'ai rien demandé. (p. 20) 
Le narrateur affirme que :

« Si un homme jurait l'aimer, elle avait fait semblant, pour retenir son corps. Elle n'éprouvait aucun sentiment. » (p. 23)

Mina est aussi superstitieuse. Pour se libérer des fantômes qui la hantaient, elle visite une devineresse martiniquaise. Celle-ci lui dit que c'était elle la chaine qui retenait Rosalia sur cette terre. Pour rompre la chaine, elle avait trois options, parmi lesquelles il y avait le retour à son pays natal. Même si elle ne voulait pas y rentrer car tout le monde était mort, elle décide d'y aller finalement. C'est là où reste la solution à son problème. Son retour en Guadeloupe c'est le dénouement.

\subsection{Melchior et Médée Monterio}

Il s'agit des parents de Mina. Melchior se marie avec Médée après la mort de sa femme des premières noces, Marie-Perle. Les deux incarnent la situation d'un mariage des contrariés, de l'amour impossible. Médée est misérable. Elle aime beaucoup son mari qui ne l'aime pas du tout. Après la naissance de l'enfant attardé, la situation devient même plus difficile, car un enfant attardé c'est l'enfant de la mère et non pas celui du père. Médée est solitaire, sans ami sauf une autre femme aussi misérable qu'elle, la Silène Couba. Les gens se moquent d'elle parce qu'elle a eu un enfant qui n'est pas normal. Elle est accusée de ne pas avoir su consoler son mari après la mort de sa femme. Suite à la naissance de sa deuxième enfant, Mina, qui est normal, sa vision de la vie change et elle apprend à s'aimer et à s'habiller. C'est une femme qui respecte son mari et n'a jamais était infidèle en dépit de tous ses problèmes. Elle donne l'image d'une femme traditionnelle, soumise.

«Las, depuis son mariage, elle dérivait dans sa propre existence, pareille à une créature bafouée par le destin. Haute négresse, elle marchait le dos voûté, (...), dans des robes qu'elle confectionnait ellemême, à la hâte, sans grand souci de plaire. (...). Médée se comportait comme ces vieilles oubliées de la mort. » (p.30)

Voici comment le narrateur la décrit. Elle est frustrée, désespérée et découragée. Ce personnage dépeint le rôle social de la femme dans la société guadeloupéenne de cette époque, à savoir, l'objet et le réceptacle des plaisirs masculins.

Melchior lui-même était même plus misérable, surtout après avoir eu un enfant attardé. D'après l'auteur, il « subissait sa vie sur terre comme le pays supportait les temps de cyclones, les avalasses dans les mornes ou la sécheresse du carême ». Melchior ne s'est marié avec "la négresse », Médée que pour remplacer sa femme qui est morte car « un homme ne peut rester seul en ce monde. » Il ne l'aimait pas. Elle était là « juste là pour cuire son manger, laver son linge, lui assurer son postérité et servir de mère à Olga ». (p. 29) Olga c'est son enfant née de son premier mariage. Médée est une esclave à 
son service, d'après les raisons qu'il donne pour son mariage à elle. Pour les deux, le sexe se fait lorsque leurs corps se touchaient sur le lit, sans parole ni caresses. Le matin, les corps séparaient, "chacun s'en va sur son route. " (p. 30)

La femme dans cette société est marginalisée et dévalorisée. Elle est tout simplement un objet de jouissance.

\subsection{Suzon Mignard}

Elle est amie de Melchior avant son premier mariage à Marie Perle. Elle est aussi sa sœur, mais elle ne le savait pas pendant des années. Melchior découvre que celle-ci c'est sa sœur seulement après avoir couché avec elle plusieurs fois. Leur relation est donc incestueuse, ce qui montre la perte de valeurs morales dans cette société. A travers ce personnage, on peut dégager la nature satirique du roman. Suzon va jusqu'à éliminer les deux femmes de Melchior pour pouvoir se marier avec lui, ne sachant pas que c'était son frère. Elle ne demande pas la raison pourquoi celui-ci ne s'est pas marier avec elle malgré le fait qu'il l'aimait bien. Sa mère, Lucinda Mignard ne le lui avait pas révélé. Elle élimine Melchior lui-même après avoir appris que c'est son frère et que le mariage serait impossible. Elle incarne la méchanceté, la jalousie et l'égocentrisme comme on peut voir ci-après.

« Suzon, elle n'a appris que trop tard que Melchior était son frère. Elle a toujours voulu se marier avec celui-ci et a visité plus de dix sorciers dans sa quête pour le mariage. Elle incarne la sorcellerie, la méchanceté et l'amour impossible. D'après le narrateur, c'est une femme méchante et jalouse qui est à l'origine des morts violentes des Monterio, orchestrées à l'aide de différents sorciers (p. 173-176), une vraie croque mitaine $\gg$.

Elle est hantée finalement par Rosalia qui apparaît dans sa maison plus de vingt ans après sa mort.

\subsection{Olga}

C'est la fille de Melchior et demi sœur de Mina. Elle n'a pas accepté le mariage de son père à Médée et ne l'a jamais appelé Mère. Elle incarne la haine et la jalousie. Elle hait sa belle mère et sa demi-sœur Rosalia. L'auteur raconte que :

Pour designer Rosalia, elle disait « elle », ne 1'appelait jamais par le nom. (...) Elle se déchainait sur elle avec ses griffes, ses poings, ses pieds.

Elle n'est pas gentille. Elle tourmente Rosalia. Après son mariage et son départ en France, elle oublie complètement sa famille. Stérile, sans enfant et malheureuse après des années de mariage à Douglas, elle invite enfin sa sœur Mina chez elle. Elle mène une vie riche. Elle est divorcée finalement. 


\section{Thématique de l'œuvre}

Le récit picaresque est caractérisé par les thèmes de l'orphelinage, l'errance, la faim, l'apparence, la violence de la mort et le destin. Nous allons dans la partie suivante essayer de dégager ces thèmes dans l'œuvre de Gisèle Pineau.

\subsection{Orphélinage}

L'orphélinage c'est le fait d'être sans parents. Manquer quelque chose pourrait être considérer comme être orphelin de cette chose. Mina affirme qu'elle fait le sexe et pas l'amour car elle n'a jamais connu l'amour. (p.12) Elle souffre du manque d'affection, d'attention. Après la mort de ses parents elle est restée avec sa sœur Rosalia, plus âgée qu'elle mais attardée. Elle devait s'occuper de celle-ci à partir de l'âge de onze ans. Les deux enfants ont mené une vie difficile. Pendant trois ans, les deux petites filles vivaient seules, dans la solitude, abandonnées, comme l'exprime la romancière ci-après :

« De rares personnes s'aventuraient à grimper à leur rencontre, pour apporter un manger tout chaud (...) Elles s'en retournaient dépitées, ne sachant trouver les mots propres à définir l'état d'abandon qui régnait sur la morne. (...) Trois années durant, elles vécurent ainsi, se suffisant de leur commune solitude et se nourrissant surtout de cassaves au coco qu'elles cherchaient le vendredi soir chez Mormone. » (p. 51)

Les deux sœurs étaient orphelines, de leurs parents, mais aussi orphelines de l'amour parental. Mina avait peur et comme elle le dit, l'un de ses préoccupations à ce moment-là c'était de veiller que la mort ne venait pas les chercher, elles aussi. Le narrateur raconte que :

«Quand Mina recevait des lettres du proviseur, elle quittait la morne au contrecœur, suppliant la Rose de l'attendre sans se désoler ni bêtiser en prenant toujours garde à la mort. » (p. 52)

Elle avait très peur de la mort qui lui a pris ses parents. Malheureusement, après trois ans, la mort est venue chercher Rosalia, mort de l'incendie quand leur case a pris feu. Mina est restée seule et traumatisée à l'âge de quatorze ans. Elle est apeurée, malheureuse et frustrée, car la maison familiale est aussi brulée. Quand un jour elle rencontre un noir, elle se dit que celui-ci faisait partie de :

« La grande famille des noirs dispersés aux quatre coins du monde. Ils partageaient en secret la douleur des tribulations du passée. »

Elle évoque tout de suite après sa situation d'orpheline :

« Je n'ai pas de famille. Je suis seule. Seule comme une île au mitan de la mer. Seule dans ma vie. Seule dans mon corps. »

Francis Assaf affirme que « Le picaro est affranchi des obligations inhérentes aux liens familiaux. Cet affranchissement se manifeste par un état 
d'orphelin. Cette autonomie précède toute mise en marche de la dynamique picaresque. »

L’orphélinage ainsi que les difficultés qu'elle a vécues permettent le voyage en France pour Mina. Elle est libre, livrée à l'errance et au commencement de ses aventures picaresques qui facilitent la continuité du récit.

\subsection{Errance}

La mobilité du héro dans un roman picaresque multiplie les espaces, comme l'affirme Daniel Marcheix, (1972), « La première chose qui frappe lorsqu'on lit un roman picaresque, c'est la mobilité du héros. Cette errance est d'abord géographique d'où la multiplicité des espaces». Le personnage principal vit dans une certaine ville ou un certain pays, dans un espace. L'espace pourrait être carcéral, (interdit), ou libre. L'héroïne du Chair Piment de Gisèle Pineau se trouve d'abord dans un espace libre, en famille en Guadeloupe où elle est adorée par son père, sa mère et sa sœur. Après la mort de ses parents, elle se trouve abandonnée et en situation de difficulté. L'auteur affirme que «Le monde qui lui était maintenant offert s'avérait cruel et austère. Restaient plus que la pauvre Rose et Olga, partie en France ». L'héroïne se trouve dans un espace cruel, carcéral. Elle voyage en France pour habiter avec sa sœur, toujours en espace interdit. Elle se trouve chez sa demisœur qui avait maltraité Rosalia, ce qui lui est très difficile pendant trois ans. Quand finalement elle trouve son propre appartement, elle est dans un espace libre, et elle peut se lancer facilement dans ses aventures picaresques.

Les lieux parcourus par le personnage principal dans le roman de Gisèle sont réels : Piment, la Guadeloupe, Paris, la France, la Martinique. Le lecteur trouve donc dans le texte des éléments extra diégétiques. Les termes employés par la romancière sont réels et peuvent être repérés par le lecteur en dehors du roman. Cela a une fonction mimésique. Il donne l'illusion de la réalité, l'impression que le récit est réel. Les espaces parcourus par les personnages révèlent les relations entre l'auteur et son monde.

Il est à noter que Gisèle Pineau est née de parents Guadeloupéens. Elle a vécu en Martinique et s'est installée à Paris en France après ses études. Il est évident qu'elle a des rapports affectifs avec les lieux mentionnés dans son texte, à savoir, la Martinique, la Guadeloupe et la France. La romancière dans ce roman s'adresse au lecteur explicite ainsi qu'au lecteur implicite. L'analyse de l'espace est importante car l'auteur s'adresse à quelqu'un dans cette société là. Son texte, c'est le reflet de la société dans laquelle se situe le récit. Pour Mina, la Guadeloupe est un pays où elle a souffert de la solitude et des traumatismes, et tout le monde là-bas est mort. 
« C'est un pays de l'inceste, d'une famille écartelée, des sorciers, de la mort: Marie-Perle noyée, Médée écrasée, Melchior foudroyée,

Rosalia mort d'un incendie. » (p. 367).

Quand elle visite la devineresse, celle-ci lui propose de rentrer à Piment pour couper la chaine qui retenait sa sœur décédée sur cette terre, elle refuse. Elle ne trouve pas de raison pour y rentrer puis qu'elle se trouve déjà dans son " univers » où elle a de la paix, où elle est libre :

«Il n'en est pas question... Jamais je ne retournerai au pays... »

« Ils sont tous morts. » (p. 16)

\subsection{Faim}

Dans le récit picaresque, l'accent est mis sur le niveau matériel de l'existence. D. Souiller, (1980), parle de «l'omniprésence du thème de l'argent comme moteur du personnage $»$. Les filles allaient chercher à manger chez mormone. Il s'agissait des cassaves à coco. Des fois elles recevaient de la nourriture chaud des bienveillants qui descendaient chez eux de temps en temps. Tout cela renvoie au thème de l'argent. Même si elles recevaient une pension qu'elles allaient chercher à la poste, ce n'était rien. Elles ont connus la faim. Elles menaient une vie dépouillée de l'amour parental (faim de l'amour), de la nourriture cuite (reçue de temps en temps des voisins), de la nourriture de l'esprit (elles n'allaient plus à l'école) et de l'âme (liberté et espoir). Elles n'étaient pas libres car elles avaient toujours peur de la mort.

\subsection{Violence de la mort}

La mort violente est présente dans le roman picaresque de sphère francophone, et dans le texte de Gisèle Pineau. Mina, l'héroïne, est hantée par la violence de la mort. Elle a perdu d'abord sa mère, suivie par son père et finalement sa sœur : toute la famille. Elle ne voulait pas parler de la mort de Rosalia parce que cela la tourmentée. Elle l'avait témoignée et voyait toujours sa sœur,

«Sa chemise de nuit brulée, ses yeux rouges, ses nattes en flammes sur la tête.» (p. 54)

Tous sont morts de façon violente : la mère écrasée par un camion, le père terrassé par la foudre et Rosalia brulée vive. Elle est restée seule. D'après le narrateur, en vivant ses aventures picaresques, Mina essayait de ne pas penser à la mort, elle en avait peur elle-même :

" Même si la peur de disparaître de façon violente, comme tous ce de sa famille, la tisonnait toujours. » (p. 94)

\subsection{Apparence, sorcellerie et le surnaturel}

Il s'agit ici du masque que portent les personnages pour cacher quelque chose. Ils présentent ce qu'ils ne sont pas ce qui est source d'illusion. La mère 
de Mina, Médée, même si elle souffrait dans son mariage portait toujours un masque, de même que son mari. Olga, elle avait deux visages, comme l'auteur nous la présente. Un visage très gentille, surtout quand elle était avec Mina, et l'autre méchante, surtout quand elle est avec Rosalia et sa belle-mère, Médée. Suzon Mignard est la cause des problèmes chez Melchior. Cependant, c'est elle qui reçoit Mina après la disparition définitive des membres de sa famille, et prétend être très gentille avec la petite. Elle porte un masque. La sorcellerie et le surnaturel sont deux autres thèmes qui se trouvent dans le roman de Gisèle Pineau. Suzon Mignard incarne la sorcellerie, la méchanceté et l'inceste. Elle est à l'origine des morts violentes chez les Monterio. Rosalia, qui hante Mina revient enfin la hanter, mais elle ne le dit à personne. Celle-ci est apparue à Piment plus de vingt ans après sa mort, et les gens disaient l'avoir rencontrée dans les rues. Quand on lui demandait si elle l'avait vue, elle disait non, et pourtant Rosalia apparaissait tout le temps dans sa maison.

\section{Forme du corpus}

\subsection{La narration}

La narration a une forme fragmentée et répétitif. Il s'agit de récits enchâssés, avec des textes y insérées qui n'ont qu'un rapport lointain avec le récit principal. La narration se fait en forme d'épisodes. Van Tieghem, (1968), écrit que, techniquement, « le roman picaresque n'a pas d'architecture solide, et [que] le lien entre les épisodes est très faible. » Il s'agit du principe de la discontinuité. Dans Chair Piment, l'histoire est composée de plusieurs autres histoires y insérées par l'auteur. Toute nouvelle personne constitue une nouvelle histoire. L'une finit, l'autre commence. La structure est donc cyclique. Le récit est contient des histoires multiples, intercalées : 1'histoire de Mina et Rosalia; la vie de mina en France; le mariage de Melchior et Médée ; Suzon et la sorcellerie ; la maladie de Victor ; les visages multiples d'Olga parmi d'autres.

L'œuvre est composée de plusieurs épisodes: Mina « dans son univers » en France ; La naissance de Mina ; La mort de ses parents ; la mort de Rosalia ; Le voyage de Mina en France ; Mina chez sa sœur et ses rapports avec son mari ; Mina de retour en Guadeloupe, etc.

Ces épisodes constituent la structure du récit. La structure qui forme le corpus de Chair Piment peut être résumée comme suit :

Quête $\longrightarrow$ Succès provisoire $\longrightarrow$ Echec $\longrightarrow$ Recommencement

Suivant la perte douloureuse de des siens, Mina part en France pour commencer une nouvelle vie (succès provisoire). Là, son problème n'est pas résolu (échec), et elle est obligé de rentrer en Guadeloupe (recommencement,) pour se libérer de sa sœur qui la hantait, et de la peur de la mort. 


\subsection{Le temps}

Le temps de l'histoire joue un rôle important dans la narration, car elle définit son rythme et fixe la nature du discours. On distingue deux types de temps dans le récit : le temps de la chose racontée (temps du signifiée), et le temps du récit (temps du signifiant). Dans cette œuvre, il y a décalage entre l'histoire (la chose racontée) et le récit (l'énoncé narratif). L'ordre de la narration ne correspond pas à l'ordre chronologique de la fiction. Il y a discordance entre l'ordre des événements de l'histoire et leur ordre dans le récit. L'auteur commence au milieu de l'histoire. Elle raconte d'abord la vie menée par Mina en France. Cette histoire est suspendue pendant un moment et l'auteur revient sur le mariage difficile de ses parents, sa naissance et son enfance. Il s'agit d'un va et vient, un avance- recule sur les aventures de Mina en France. Cela se fait pour installer la tension chez le lecteur, le perturber et susciter les anticipations. L'auteur nous montre les effets (le comportement bizarre de Mina), avant de parler des causes. Ce va et vient permet de combler les déficits d'informations provoqués par les anticipations.

Le temps du récit est éclaté. L'auteur passe du futur proche à l'imparfait, au présent, au passé composé, puis au passé simple.

« Tu vas partir maintenant ? » Avait murmuré Mina. « Tu es contente ?

$\mathrm{Tu}$ as eu ce que tu voulais. C'est fini. Tu vas me laisser... » (Futur proche + imparfait+ présent+ passé composé + futur proche)

Rosalia secoua la tête. Des flammèches éclaboussèrent les meubles de la chambre. (Passé simple employé par le narrateur.)

« Je ne veux plus te voir. Tu es méchante ! Tu es un démon. Olga avait bien raison de te frapper... » (Le présent + l'imparfait).

Rosalia se rapprocha du lit. (Passé simple employé par le narrateur.) (p. 84)

L'éclatement de temps lui permet de faire la distinction entre celui qui parle, ce qu'il dit et ce qu'il pense ainsi que le temps exact du discours.

\section{Conclusion}

L'objectif de ce travail était d'analyser le texte de Gisèle Pineau pour voir en quoi il est picaresque. Nous trouvons que le personnage principal, Mina Monterio pourrait être qualifiée de picaro. Faire le sexe avec n'importe qui gratuitement c'est sa préoccupation. Elle est déraisonnable et ne respecte pas les valeurs sociales. Elle essaie de sortir de sa situation difficile en employant des moyens inacceptables. C'est une femme rebelle qui souffre de la pauvreté intellectuelle et qui fait des choses qu'une femme normale n'oserait pas faire. C'est de la folie.

La société décrite par Gisèle dans Chair piment est composée de noirs, des antillais, descendants des anciens esclaves mais aussi des blancs. L'auteur peint des gens qui souffrent toujours des effets de la discrimination, des 
relations noirs/blancs. C'est une société en crise, caractérisée par l'adultère, l'inceste, la sorcellerie et la manque de tolérance. Le surnaturel y existe aussi. Ce sont les gens qui ne sont pas sages. Il s'agit d'une société pic arisée dont les habitants ne sont pas sages.

Suzon Mignard fait recours à la sorcellerie pour forcer un mariage impossible avec son « frère » Melchior et elle échoue trois fois. Elle n'est pas intelligente. Elle devrait se demander pour quoi Melchior a brusquement changer d'avis et a refusé de se marier avec elle. Elle apprend après des années que c'est son frère. La sagesse n'existe pas chez elle.

Gisèle raconte la vie misérable de Mina Monterio, sa naissance, ses problèmes, son voyage en France, ses aventures picaresques et son retour au pays natal. Les allers-retours dans l'histoire sont caractéristiques du roman picaresque.

Finalement, il faut noter la portée didactique de l'œuvre, les leçons que le lecteur pourrait tirer de l'histoire. Gisèle Pineau voudrait montrer, à travers le récit que les gens n'apprennent pas de leçons de leur passé. Les mêmes erreurs se répètent. Dans Chair Piment, les relations incestueux, la sorcellerie, les morts violentes, l'adultère et l'amour misérable sont tous à cause des erreurs qui se répètent. Le roman est le reflet d'une société instable, en quête de la paix. Une société caractérisée par des relations conflictuelles, des amours malheureux et de la sorcellerie. Dans ce roman, les erreurs sont dévoilées à la fin, et la victoire du bien sur les vices est affirmé.

\section{References:}

1. Alter, R., (1964): 'Rogue's Progress: Studies in the Picaresque Novel', No. 26 of Harvard Studies in Comparative Literature, Cambridge, MA: Harvard University Press

2. Assaf, F., (1983), Lesage et le picaresque, Paris, Nizet, cité par Bodo Bidy Cyprien.

3. Christensen, J, (1994). "Don Picaro: Lord Byron and the Reclassification of the Picaresque", in The Picaresque: A Symposium on the Rogue's Tale, edited by Carmen Benito Vessels and

4. Michael O. Zappala, London; Associated University Press

5. Daniel W., (1976), The picaresque in African fiction, in Bashra, no.7

6. Devitt, A. J., (2004): Writing Genres. Rhetorical Philosophy and Theory. Carbondale, IL: Illinois University Press

7. Marcheix, D., (1972), Permanence et renouveau du picaresque dans la littérature français du XXe siècle, mémoire de maitrise, Université de Limoges.

8. Souiller D., (1980), Le roman picaresque, Paris, P.U.F cité par Bodo Bidy Cyprien 
9. Tiegem, V., (1968), Dictionnaire de littérature, vol 3, Paris, P.U.F cité par Bodo Bidy Cyprien.

10. Bodo, C. B., 2005, Le picaresque dans le roman africain sub-saharien d'expression française, Thèse de doctorat, Université de Limoges (en ligne) $[2 / 12 / 2016]$ 